\title{
$\mathrm{C}-\mathrm{A} / \mathrm{AP} / \# 122$
}

November 2003

\section{Simulation of Proton Therapy Treatment Verification via PET imaging of Induced Positron-Emitters}

J. Beebe-Wang, P. Vaska, F.A. Dilmanian, S.G. Peggs and D.J. Schlyer

"This paper was submitted to the Conference Record for the

IEEE Nuclear Science Symposium/Medical Imaging Conference October 2003."

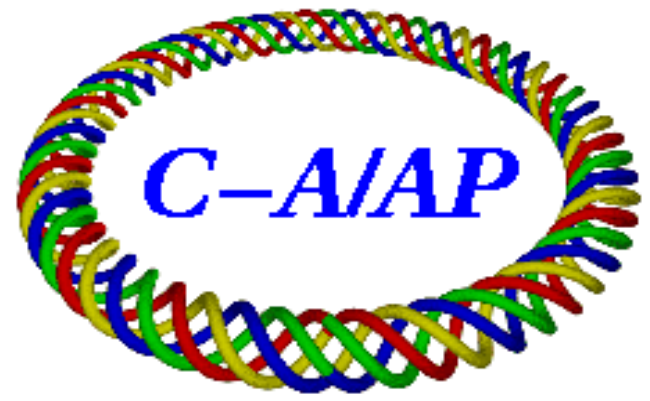

Collider-Accelerator Department

Brookhaven National Laboratory

Upton, NY 11973 


\title{
Simulation of Proton Therapy Treatment Verification via PET Imaging of Induced Positron-Emitters*
}

\author{
J. Beebe-Wang, P. Vaska, F. A. Dilmanian, S. G. Peggs and D. J. Schlyer \\ Brookhaven National Laboratory, Upton, NY 11973, USA
}

\begin{abstract}
Earlier works, including a recent one at BNL, demonstrated that PET is a promising technique to verify the dose distribution of proton therapy, which is increasingly used in radiation oncology because the dose conforms more tightly to the tumor than common $x$-ray radiation therapy. Proton therapy produces positron-emitting isotopes along the beam path, allowing the therapy dose distribution to be imaged by PET as a form of quality assurance of the treatment. This is especially important when treating inhomogeneous organs such as the lungs or the head-and-neck, where the calculation of the expected dose distribution for treatment planning is more difficult. In this paper, we present Monte Carlo simulations of the yield of positron emitters produced by proton beams up to $250 \mathrm{MeV}$, followed by statistically realistic Monte Carlo simulation of the images expected from a clinical PET scanner. The emphasis of this study is to accurately predict the positron emitter distribution and to determine the quality of the PET signal in the region near the Bragg peak which is critical to the success of PET imaging for verification of proton beam location and dosimetry. In this paper, we also demonstrate that the image results depend strongly on the available nuclear reaction cross section data. We determine quantitatively the differences in the calculated positron emitter yields resulting from four different sets of input nuclear reaction cross section data. They are compared to the simulated distributions of positron emitter productions and absorbed proton energies.
\end{abstract}

\section{INTRODUCTION}

Positron emission tomography (PET) is potentially a very useful tool for monitoring the distribution of the dose deposited in the patient from proton therapy [1]-[8]. This method is based on the detection of the positron-annihilation $\gamma$ rays following the decay of the small amounts of positron emitters (typically ${ }^{11} \mathrm{C},{ }^{13} \mathrm{~N}$ and ${ }^{15} \mathrm{O}$ ) produced via non-elastic nuclear reaction of protons with the target nuclei of the irradiated tissue. Verification of the therapy can be achieved by comparing the PET images discerning the positron activity distribution with the predicted target dose distribution used to plan the treatment. The PET image essentially displays the inverse of the deposited energy distribution because the nonelastic nuclear reaction cross sections provide signal along the beam path, but diminish at the Bragg peak, where most of the proton energy is deposited via other interactions. However, an effective dose verification can still be made by comparing the radioisotope distribution measured by PET with the yield of the positron emitters predicted from the treatment planning code.

*Work performed under the auspices of the US DOE.
The possibility of proton therapy monitoring by means of PET was investigated by various groups [2]-[8]. However, due to the limitations of available non-elastic nuclear cross section data and detailed simulation codes, most of the simulation studies carried out in the past did not address the issue of the low energy end of the proton track, which is essential in monitoring the Bragg peak. In this paper, we examine the potential of PET as a quality assurance method for the full proton energy range $(0.1$ to $250 \mathrm{MeV})$ and determine the quality of the PET signal in the region near the Bragg peak which is critical to the success of PET imaging for verification of proton beam location and dosimetry. The incentive for this work was the design of the Rapid Cycling Medical Synchrotron (RCMS) [9] at Brookhaven National Laboratory.

\section{POSITRON EMITTER PRODUCTION}

During proton therapy, even though many isotopes are produced through different nuclear interactions, there are only six major channels producing the positron emitters ${ }^{11} \mathrm{C},{ }^{13} \mathrm{~N}$ and ${ }^{15} \mathrm{O}$ in human tissue as listed in TABLE I.

TABLE I

RELEVANT POSITRON-EMITTER PRODUCTION REACTIONS

\begin{tabular}{cccc}
\hline $\begin{array}{c}\text { Nuclear } \\
\text { Reactions }\end{array}$ & $\begin{array}{c}\text { Threshold } \\
\text { Energy } \\
(\mathrm{MeV})\end{array}$ & $\begin{array}{c}\text { Half-life } \\
\text { Time } \\
(\mathrm{min})\end{array}$ & $\begin{array}{c}\text { Positron } \\
\text { Max. Energy } \\
(\mathrm{MeV})\end{array}$ \\
\hline${ }^{16} \mathrm{O}(\mathrm{p}, \mathrm{pn}){ }^{15} \mathrm{O}$ & 16.79 & 2.037 & 1.72 \\
${ }^{16} \mathrm{O}(\mathrm{p}, 2 \mathrm{p} 2 \mathrm{n}){ }^{13} \mathrm{~N}^{\mathrm{a})}$ & $5.66^{\mathrm{c})}$ & 9.965 & 1.19 \\
${ }^{14} \mathrm{~N}(\mathrm{p}, \mathrm{pn}){ }^{13} \mathrm{~N}$ & 11.44 & 9.965 & 1.19 \\
${ }^{12} \mathrm{C}(\mathrm{p}, \mathrm{pn}){ }^{11} \mathrm{C}$ & 20.61 & 20.39 & 0.96 \\
${ }^{14} \mathrm{~N}(\mathrm{p}, 2 \mathrm{p} 2 \mathrm{n}){ }^{11} \mathrm{C}^{\text {a) }}$ & $3.22^{\mathrm{c})}$ & 20.39 & 0.96 \\
${ }^{16} \mathrm{O}(\mathrm{p}, 3 \mathrm{p} 3 \mathrm{n}){ }^{11} \mathrm{C}^{\mathrm{b})}$ & $27.50^{\mathrm{c})}$ & 20.39 & 0.96 \\
\hline
\end{tabular}

a): $(p, 2 p 2 n)$ is inclusive of $(p, \alpha)$

b): $(p, 3 p 3 n)$ is inclusive of (p, $\alpha \mathrm{pn})$

c): The listed thresholds refer to $(p, \alpha)$ and $(p, \alpha p n)$

TABLE II summarizes the other 15 more exotic nuclear reactions leading to the production of positron emitters. Since ${ }^{13} \mathrm{C},{ }^{15} \mathrm{~N}$ and ${ }^{18} \mathrm{O}$ have very low abundances in the human body, the reactions induced by protons with these isotopes are negligible. The cross sections of the radioactive capture reactions, $(p, \gamma)$, are typically micro-barns, or three orders of magnitude smaller than the six main channels listed in TABLE I. Also, due to the very small quantities of production of ${ }^{10} \mathrm{C}$ and ${ }^{14} \mathrm{O}$ isotopes, the uncertainties in their cross section data would make the calculation results meaningless. Therefore, the reactions listed in TABLE II are excluded in this study. 
TABLE II

LOW PROBABILITY REACTIONS THAT PRODUCE POSITRONEMITTING NUCLEI

\begin{tabular}{cccc}
\hline $\begin{array}{c}\text { Nuclear } \\
\text { Reactions }\end{array}$ & $\begin{array}{c}\text { Threshold } \\
\text { Energy } \\
(\mathrm{MeV})\end{array}$ & $\begin{array}{c}\text { Half-life } \\
\text { Time } \\
(\mathrm{min})\end{array}$ & $\begin{array}{c}\text { Positron Max. } \\
\text { Energy } \\
(\mathrm{MeV})\end{array}$ \\
\hline${ }^{12} \mathrm{C}(\mathrm{p}, \mathrm{p} 2 \mathrm{n}){ }^{10} \mathrm{C}$ & 34.5 & 0.32 & 1.87 \\
${ }^{12} \mathrm{C}(\mathrm{p}, \gamma){ }^{13} \mathrm{~N}$ & 0 & 9.97 & 1.19 \\
${ }^{13} \mathrm{C}(\mathrm{p}, \mathrm{p} 2 \mathrm{n}){ }^{11} \mathrm{C}$ & 25.5 & 20.3 & 0.96 \\
${ }^{13} \mathrm{C}(\mathrm{p}, \mathrm{n}){ }^{13} \mathrm{~N}$ & 3.2 & 9.97 & 1.19 \\
$\left.{ }^{14} \mathrm{~N}(\mathrm{p}, \mathrm{n} \alpha)\right)^{10} \mathrm{C}$ & 17.2 & 0.32 & 1.87 \\
${ }^{14} \mathrm{~N}(\mathrm{p}, \gamma){ }^{15} \mathrm{O}$ & 0 & 2.04 & 1.72 \\
${ }^{14} \mathrm{~N}(\mathrm{p}, \mathrm{n}){ }^{14} \mathrm{O}$ & 6.6 & 1.18 & 1.81 \\
${ }^{15} \mathrm{~N}(\mathrm{p}, \mathrm{n} \alpha)^{11} \mathrm{C}$ & 14.7 & 20.3 & 0.96 \\
${ }^{15} \mathrm{~N}(\mathrm{p}, \mathrm{nd})^{13} \mathrm{~N}$ & 20.4 & 9.97 & 1.19 \\
${ }^{15} \mathrm{~N}(\mathrm{p}, \mathrm{t}){ }^{13} \mathrm{~N}$ & 13.8 & 9.97 & 1.19 \\
${ }^{15} \mathrm{~N}(\mathrm{p}, \mathrm{n}){ }^{15} \mathrm{O}$ & 3.8 & 2.04 & 1.72 \\
${ }^{16} \mathrm{O}(\mathrm{p}, \gamma){ }^{17} \mathrm{~F}$ & 0 & 1.07 & 1.74 \\
${ }^{16} \mathrm{O}(\mathrm{p}, 3 \mathrm{p} 4 \mathrm{n}){ }^{10} \mathrm{C}$ & 39.1 & 0.32 & 1.87 \\
${ }^{16} \mathrm{O}(\mathrm{p}, \mathrm{p} 2 \mathrm{n}){ }^{14} \mathrm{O}$ & 30.7 & 1.18 & 1.81 \\
${ }^{18} \mathrm{O}(\mathrm{p}, \mathrm{n}){ }^{18} \mathrm{~F}$ & 2.6 & 109.8 & 0.64 \\
\hline
\end{tabular}

\section{NUCLEAR REACTION CROSS SECTIONS}

The expected number of nuclear reactions is governed by three factors: nuclear reaction cross sections, the number of incoming particles limited by target dose, and the number of target particles. If the incoming proton beam flux and target particle density are fixed, the positron emitter production is determined by the cross section data used in the simulations. Therefore, reliable cross section data is essential to the prediction of positron emitter production. However, the currently available data are very limited -- either incomplete or un-compiled -- because very few experimental measurements have been performed on these reactions especially in the low energy range below $50 \mathrm{MeV}$. In this study, we use four sets of available nuclear reaction cross section data for comparison:

1. Data extracted from the emission spectra of recoils in the ENDF electronic file provided by the ICRU Report 63 [10] used by J. Beebe-Wang et al. [1];

2. Data from "Experimental Nuclear Reaction Data File (EXFOR)" maintained by National Nuclear Data Center at BNL [11] used by K. Parodi et al. [6];

3. Data from TERA 95/19 TRA15 [12] used by A. Del Guerra et al. [4];

4. Data from 8 different resources during 1962-1996 collected by D. Litzenberg in Ph.D. dissertation [8].

The cross section data of the six main channels amongst these four sources are displayed and compare in Figures 1-6. In the higher energy range, where some data are not available, extrapolation was used to extend the data to $250 \mathrm{MeV}$.

The number of particles - protons and interaction products delivered to the end of proton beam track is governed by the initial proton beam flux and the probabilities of proton interactions with nuclei. The total non-elastic nuclear cross sections for protons incident on ${ }^{16} \mathrm{O},{ }^{14} \mathrm{O}$ and ${ }^{11} \mathrm{C}$ are important to this study, for example because the proton flux is attenuated. Total non-elastic cross sections are also especially important in treatment planning, since the accuracy of dose prediction in the target volume (tumor) is limited by their accuracy [13]. The total non-elastic cross section data for protons on ${ }^{6} \mathrm{O},{ }^{\mathrm{O}} \mathrm{O}$ and ${ }^{11} \mathrm{C}$ used in the simulation part of this study are presented in Figure 7 as functions of proton kinetic energy[10].

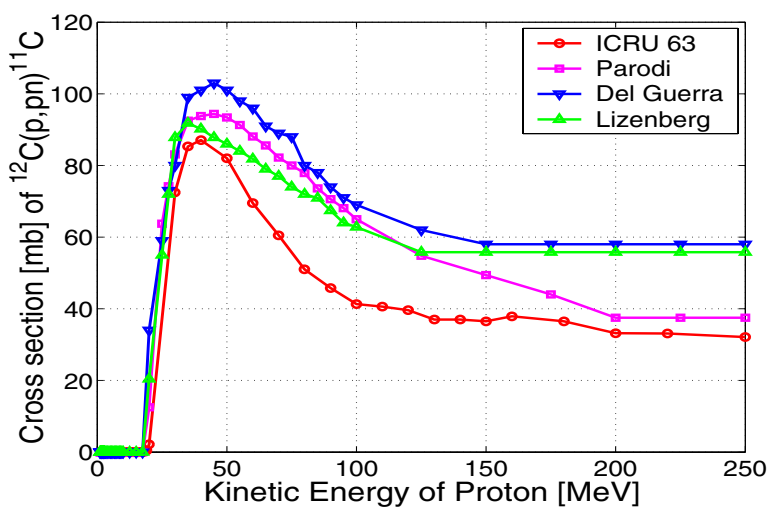

Fig. 1 Nuclear reaction cross sections of ${ }^{12} \mathrm{C}(\mathrm{p}, \mathrm{pn}){ }^{11} \mathrm{C}$. The data from four different resources are presented for comparison.

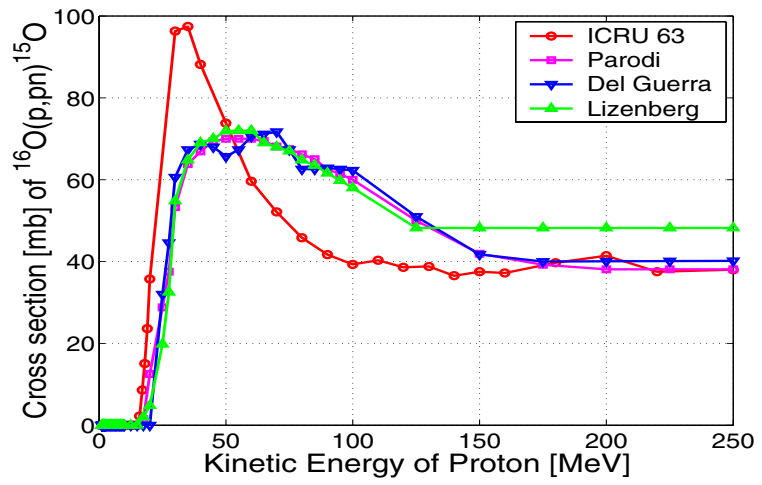

Fig. 2. Nuclear reaction cross sections of ${ }^{16} \mathrm{O}(\mathrm{p}, \mathrm{pn})^{15} \mathrm{O}$. The data from four different resources are presented for comparison.

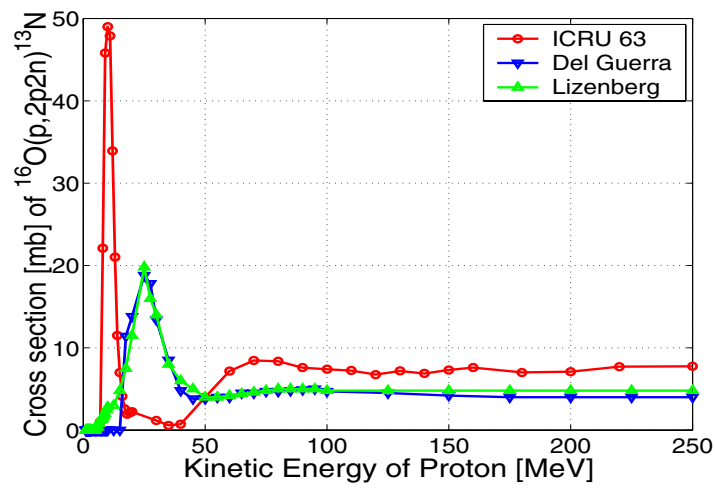

Fig. 3. Nuclear reaction cross section of ${ }^{16} \mathrm{O}(\mathrm{p}, 2 \mathrm{p} 2 \mathrm{n})^{13} \mathrm{~N}$. The data from three different resources are presented for comparison. 


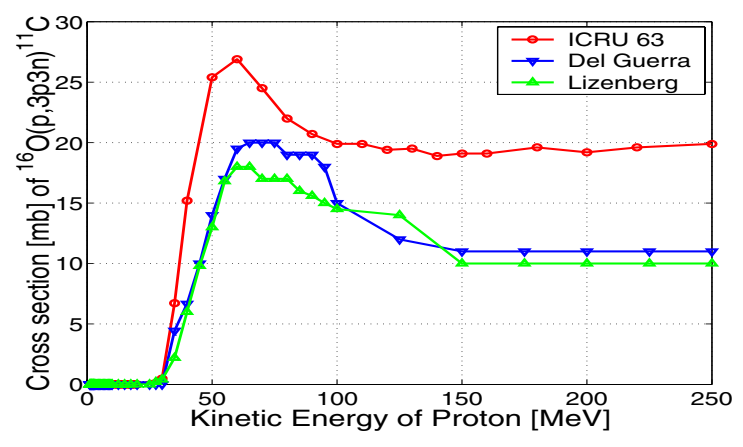

Fig. 4. Nuclear reaction cross section of ${ }^{16} \mathrm{O}(\mathrm{p}, 3 \mathrm{p} 3 \mathrm{n}){ }^{11} \mathrm{C}$. The data from three different resources are presented for comparison.

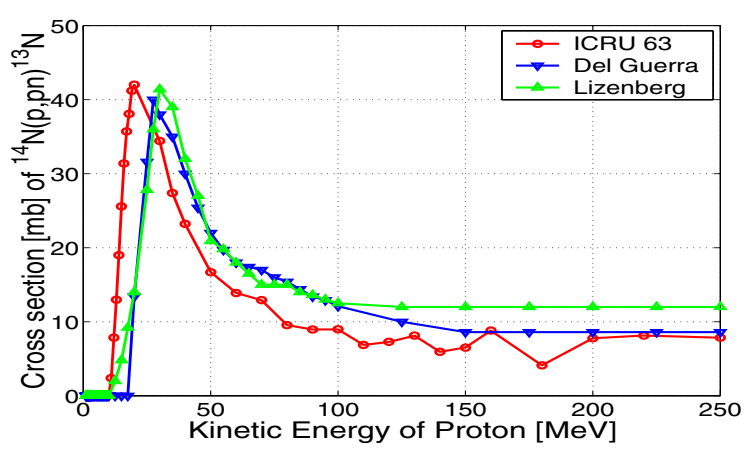

Fig. 5. Nuclear reaction cross section of ${ }^{14} \mathrm{~N}(\mathrm{p}, \mathrm{pn})^{13} \mathrm{~N}$. The data from three different resources are presented for comparison.

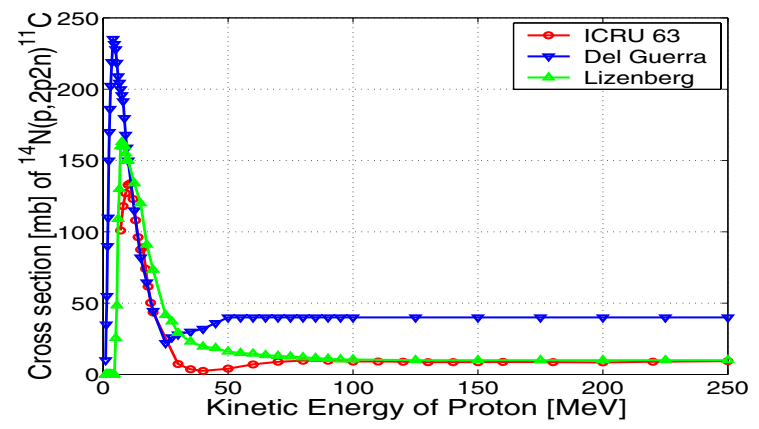

Fig. 6. Nuclear reaction cross section of ${ }^{14} \mathrm{~N}(\mathrm{p}, 2 \mathrm{p} 2 \mathrm{n}){ }^{11} \mathrm{C}$. The data from three different resources are presented for comparison.

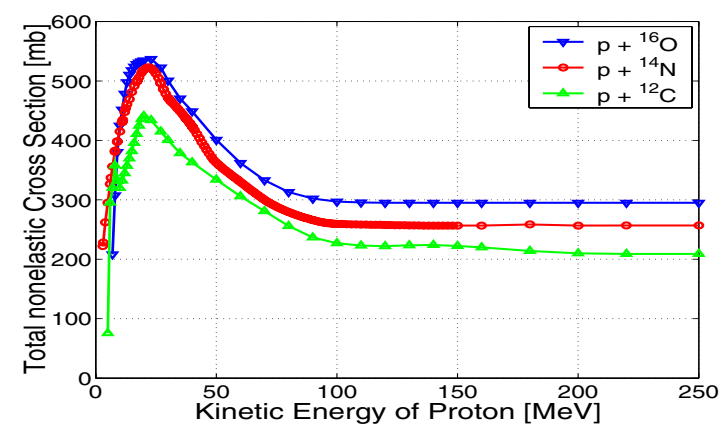

Fig. 7. Total non-elastic nuclear reaction cross sections for protons incident on ${ }^{6} \mathrm{O},{ }^{14} \mathrm{~N}$ and ${ }^{11} \mathrm{C}$ as functions of kinetic energy [10].

\section{MONTE CARLO SIMULATION}

The SRNA-BNL software package was used in this study. It was originally developed by R. D. Ilic (SRNA-2KG) [14], and was modified in BNL for this work to include also the production of positron emitter nuclei. SRNA-2KG is a Monte Carlo code for use in proton transport, radiotherapy, and dosimetry. Protons within an energy range of $100 \mathrm{keV}$ to 250 $\mathrm{MeV}$ with pre-specified spectra are transported in a 3Dgeometry through material zones confined by planes and second order surfaces. SRNA-2KG can treat proton transport in 279 different kinds of materials including elements from $Z=1$ to $Z=98$ and 181 compounds and mixtures.

The simulation of proton transport is based on the multiple scattering theory of charged particles and on a model for compound nucleus decay after proton absorption in non-elastic nuclear interactions. For each part of the range, an average loss of energy [15] is calculated with a fluctuation from Vavilov's distribution and with Schulek's correction [9]. The deflection angle of protons is sampled from Moliere's distribution [9]. SRNA-2KG have been benchmarked with the well know programs GEANT-3 [16] and PETRA [17]. Very good agreement was reached under the same conditions. Figure 8 shows the results comparison of a $250 \mathrm{MeV}$ proton pencil beam in water phantom from SRNA-2KG and GEANT3 .

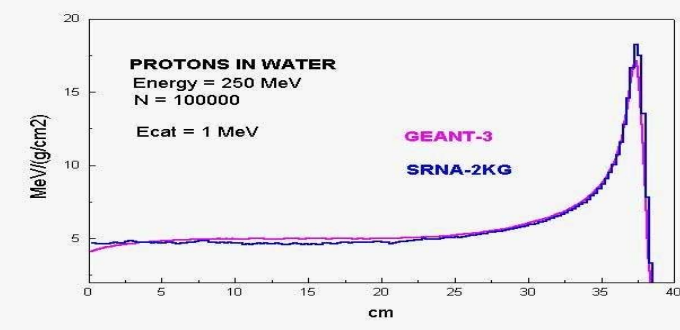

Fig. 8. Comparison of simulation results obtained from SRNA-2KG and GENT-3 (Courtesy of Dr. R.D. Ilic).

The positron emitters ${ }^{11} \mathrm{C},{ }^{13} \mathrm{~N}$ and ${ }^{15} \mathrm{O}$ are created through the decay processes of compound nuclei which include emission of protons, deuterons, tritons, alpha particles or photons. The decay products are sampled using Poisson's distribution with appropriate average multiplication factors for each particle. Energy and angle of particle emissions, and the multiplication factors are obtained from comparing the direct cross sections available for reaching the daughter nuclei with that from the integration of differential cross sections for nonelastic nuclear interactions (see figures 1-6). Energy and angle of secondary neutron emission are sampled from emission spectra. Transport of secondary protons follows that of primary protons of that particular energy. Spatial location and angle of neutron and photon are recorded, but not further treated. Emitted deuterons, tritons and alpha particles are assumed to be absorbed at the location of their creation.

In order to assess the feasibility of effectively imaging the resulting positron emitter distribution, a realistic PET scan was then simulated using the SimSET Monte Carlo PET scanner simulation package [18]. SimSET handles the most important aspects of the image formation process, including photon attenuation and scatter, geometry and photon acceptance of the tomograph, and binning of the coincidence data. For this study, modifications were made in BNL in order to 
accommodate a block detector layout, as well as standard 3Dsinogram binning. The clinical whole-body CTI HR+ tomograph was simulated with the proton beam direction aligned with the scanner axis. The attenuation of a typical human head (ellipse with axes of $15 \mathrm{~cm} \times 18 \mathrm{~cm}$ in the transaxial planes) was used to provide more realistic statistics. The output sinograms were reconstructed into volumetric images using the standard filtered back-projection technique.

\section{RESULTS}

The study is for a typical absorbed dose (2 Gray) in a typical target volume ( $5 \mathrm{~cm}$ diameter). It is achieved by modulating kinetic energies of five beam pulses so that the Bragg peaks are located $1 \mathrm{~cm}$ apart in depth. The relative beam intensities are $1.0,0.39,0.31,0.24$ and 0.22 from the highest to the lowest energy pulse. Proton beams with kinetic energies up to 250 $\mathrm{MeV}$ with $2 \mathrm{~mm}$ diameter and a zero angle of divergence were transported in a human tissue using the SRNA-BNL simulation code. The soft tissue (ICRU 4-component) used in the simulation had a 0.55 ratio of the averaged atomic number to atomic mass $(Z / A)$, and a density of $1.0 \mathrm{~g} / \mathrm{cm}^{3}$. The elemental composition of the tissue was $10.11 \%$ hydrogen, $11.11 \%$ carbon, $2.60 \%$ nitrogen, and $76.18 \%$ oxygen. The number of protons estimated to produce an absorbed dose of 2 Gray at the Bragg peak of a single pulse is $2 \times 10^{7}$.

The positron emitter spatial distributions were simulated with the cross-sections data from ICRU Report 63 [10], shown by red curves in Figures 1-6, since these data are more resent and more complete in the low energy range. In order to reduce the random noise, the values are obtained from averaging 45 sets of simulation data. The results of linear production densities of ${ }^{15} \mathrm{O},{ }^{13} \mathrm{~N}$ and ${ }^{11} \mathrm{C}$ are presented in Fig. 9, 10 and 11, respectively. The energy absorbed by the tissue is superimposed with a right-side vertical scale in the same figures for depth comparison. These positron emitter distributions are then used as the inputs to obtain the subsequently PET images with the SimSET Monte Carlo tomograph simulation.

Fig. 12 is a coronal slice from the reconstructed PET image. Despite only about 14000 coincidence counts in the entire image, the narrow transaxial distribution and lack of background activity gives sufficient contrast to provide a reasonable definition of the distribution. The expected PET image activity signal (red curve) and its standard deviation (green curves) are determined with the data extracted from the 100 sets of PET image, as shown in Figure 13. The "spreadout Bragg peak" (black curve), created by five proton beam pulses (thin black curves), is superimposed with a right-side vertical scale in figure 13 for depth comparison. In this study, a simply algorithm of polynomial curve fit was developed to analyze each set of noisy PET data. It is easy to see from the Figure 13 that, with the help of this simple software, the average value of processed data (blue curve) coincident with the expected PET image activity (red curve) with much smaller standard deviation (blue error bars) compared to unprocessed data (green curves). Depth at half maximum of PET image activity distribution is then determined to be $6.3 \mathrm{~mm}$ from the end of the "spread-out Bragg peak" in the soft tissue with 1 mm accuracy.

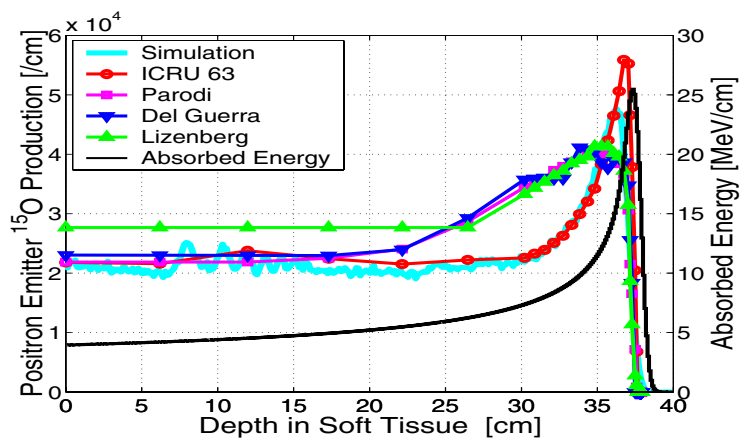

Fig. 9. The estimated production of $\mathrm{O}^{15}$ isotopes during a single pulse proton therapy session. The distributions from the simulation and the calculations with four different cross section data resources are presented.

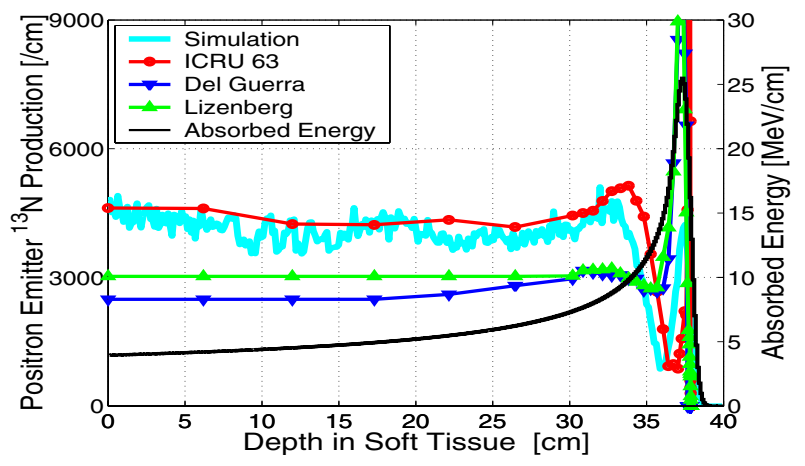

Fig. 10. The estimated production of $\mathrm{N}^{13}$ isotopes during a single pulse proton therapy session. The distributions from the simulation and the calculations with four different cross section data resources are presented.

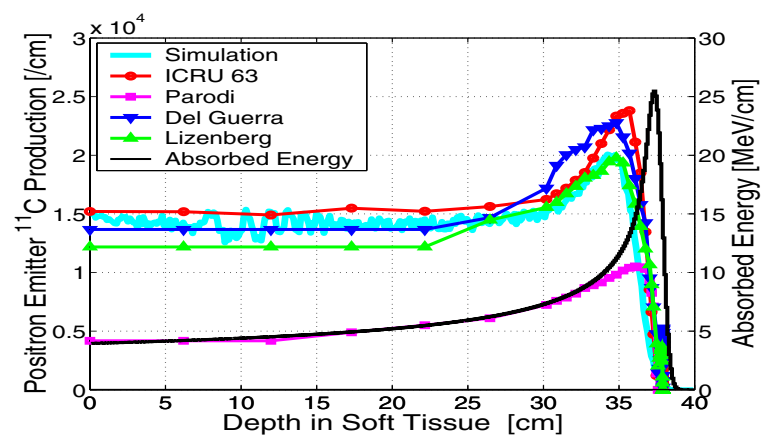

Fig. 11. The estimated production of $\mathrm{C}^{11}$ isotopes during a single pulse proton therapy session. The distributions from the simulation and the calculations with four different cross section data resources are presented.

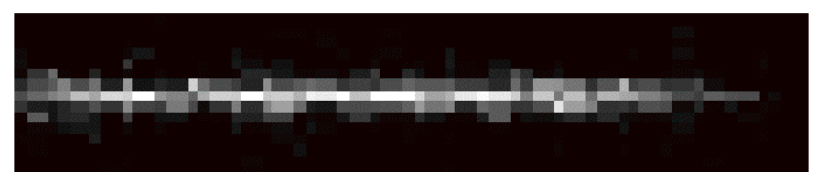

Fig.12. A $1.7 \mathrm{~mm}$ thick slice through the activity distribution of the 3-D PET image. The beam entered from left. Horizontal (axial) dimension is $15.5 \mathrm{~cm}$ (full scanner FOV). Pixel size is $2.4 \mathrm{~mm}(\mathrm{H})$ by $1.7 \mathrm{~mm}(\mathrm{~V})$. 


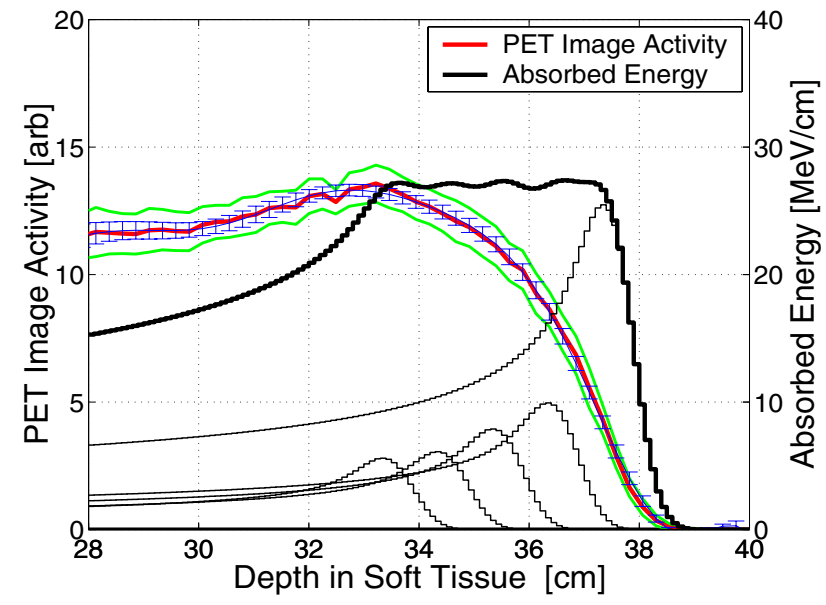

Fig.13 Depth distribution of induced activity as determined from PET image compared to the absorbed energy.

\section{DISCUSSION AND CONCLUSION}

This study demonstrate that for a typical absorbed dose (2 Gray) in a typical target volume (5 $\mathrm{cm}$ diameter) during a proton therapy session, a subsequently acquired PET image can have sufficient signal-to-noise ratio to determine the depth of the Bragg peak to $1 \mathrm{~mm}$ accuracy. We have also demonstrated, with a simply algorithm of polynomial curve fit, that a PET image data-analysis software can dramatically increase the prediction accuracy on the depth of Bragg peak. For future clinical application, the development of a more advanced robust algorithm for fitting the PET data in the presence of noise is required. The ultimate goal is comparing the radioisotope distribution measured by PET with the yield of the positron emitters predicted from the treatment planning code. Matching of these two images implies that the treatment was according to the Plan. For treatment involving multiple ports including some opposing angles the centroid of the target dose can be computed with that of the PET image.

The differences in positron emitter production distributions due to the different resources of cross section data are also demonstrated in this study. In the depth under $30 \mathrm{~cm}$ the production of the isotopes is almost without structure, reflecting the fact that the cross sections remain almost unchanged in the energy range above $100 \mathrm{MeV}$. In this range, the yields calculated with four different cross section data resources reach fairly good agreement with each other, except the ${ }^{11} \mathrm{C}$ isotope production obtained with the data from reference [11] where the ${ }^{11} \mathrm{C}$ production is only about $30 \%$ of productions obtained with the other data resources, mainly because only one of the three major channels producing ${ }^{11} \mathrm{C}$ isotope is taken into account [6]. In the depth range between $30 \mathrm{~cm}$ and $38 \mathrm{~cm}$ where the Bragg peak is located, the calculated productions are significantly different for all three isotopes (see Figures 9-11). The high yield of ${ }^{13} \mathrm{~N}$ and ${ }^{15} \mathrm{O}$ calculated with data from ICRU 63 report [10] is credited to the cross section data of reactions ${ }^{16} \mathrm{O}(\mathrm{p}, 2 \mathrm{p} 2 \mathrm{n}){ }^{13} \mathrm{~N}$ and ${ }^{16} \mathrm{O}(\mathrm{p}$, $\mathrm{pn}){ }^{15} \mathrm{O}$ in the low energy range which only became available during the year 2000 [10].
As mentioned in Section III, the total non-elastic nuclear reaction cross sections for protons incident on ${ }^{16} \mathrm{O},{ }^{14} \mathrm{O}$ and ${ }^{11} \mathrm{C}$ are important to this study and to the treatment planning, since the accuracy of dose prediction in the target volume (tumor) is limited by their accuracy [13].

This investigation shows that there is an on going need to develop a library of accurate cross section data for proton and neutron-induced reactions on the elements in human tissue. A reliable simulation or calculation depends upon accurate cross section data, especially for the investigations in the region near the Bragg peak where accurate cross section data in the low energy range (below $50 \mathrm{MeV}$ ) are needed. This is critical to the success of PET imaging for verification of the Bragg peak location of the proton beam, and dosimetry.

\section{ACKNOWLEDGMENT}

The authors would like to think Dr. R.D. Ilic and the Imaging Research Lab at University Washington for providing SRNA-2KG and SimSET software packages.

\section{REFERENCES}

[1] J. Beebe-Wang, F.A. Dilmanian, S. Peggs, D.J. Schlyer, P. Vaska "Feasibility of PET of Dose Distribution in Proton Beam Cancer Therapy", Proc. of EPAC 2002, pp.2721, (2002).

[2] W. Enghardt et al., "Positron emission tomography for quality assurance of cancer therapy with light ion beams", Nucl. Phys. A654, p.1047c, (1999).

[3] K. Parodi, W. Enghardt, "Potential application of PET in quality assurance of proton therapy", Phys. Med. Biol. 45, N151, (2000).

[4] Del Guerra, A., Di Domenico, G., "Positron Emission Tomography as an aid to in 'vivo' dosimetry for proton radiotherapy: a Monte Carlo simulation," TERA 93/10 TRA 9, August 1993.

[5] U. Oelfke et al., "Proton dose monitoring with PET: quantitative studies in Lucite", Phys. Med. Biol. 41, p.177, (1996).

[6] K. Parodi, W. Enghardt, and T. Haberer, "In-beam PET measurements of $\beta+$ radioactivity induced by proton beams", Phys. Med. Biol. 47 p.21$26(2002)$

[7] A.M.J. Paans and J.M. Schippers, "Proton Therapy in combination with PET as monitor: A feasibility study", IEEE Trans. Nucl. Sci., 40, p.1041-1043, (1993).

[8] Dale Litzenberg. "On-line Monitoring and PET Imaging of the PositronEmitting Activity Created in Tissue by Proton Radiotherapy Beams." Ph.D. Thesis, Univ. of Michigan (1997).

[9] S. Peggs et al, "The Rapid Cycling Medical Synchrotron, RCMS", Proc. of EPAC 2002, (2002).

[10] ICRU Report 63, "Nuclear Data for Neutron and Proton Radiotherapy and for Radiation", MD, USA (2000).

[11] "Experimental Nuclear Reaction Data File." Energy Sciences and Technology Dept., BNL, http://www.nndc.bnl.gov/nndc/exfor/

[12] D. Mukhopadhyay, A. Del Guerra, and O. Di Domenico, "For PETmonitored hadrontherapy", TERA 95/19 TRA15 (1995).

[13] A. Rosenfeld, Private communication, Univ. of Wollongong, Australia, (2003).

[14] R.D. Ilic, "SRNA-2KG - Proton Transport Simulation by Monte Carlo Techniques", Institute of Nuclear Sciences Vinca, Phys. Lab., (010), Beograd, Yugoslavia, February, (2002).

[15] ICRU Report 49, "Stopping Power and Ranges for Protons and Alpha Particles", MD, USA (1993).

[16] GEANT 1994 CERN Program Library W5013, (1994).

[17] J. Medin, "Studies of Clinical Proton Dosimetry Using Monte Carlo Simulation and Experimental Techniques", Ph.D. thesis, Stockholm Univ., (1997).

[18] R.L. Harrison et al., "Slat collimation and cylindrical detectors for PET simulations using simSET", IEEE Nuclear Science Symposium Conf. Record, vol.3, p.20/89- 20/92 (2000). 